\title{
Germline multigene panel testing revealed a BRCA2 pathogenic variant in a patient with suspected Lynch syndrome
}

\author{
Tomoko Yoshihama $^{1} \cdot$ Akira Hirasawa $^{1,2,3}$ (1) $\cdot$ Kokichi Sugano $^{3,4,5} \cdot$ Teruhiko Yoshida $^{5} \cdot$ Mineko Ushiama $^{5} \cdot$ Arisa Ueki $^{3}$. \\ Tomoko Akahane ${ }^{1} \cdot$ Yoshiko Nanki $^{1} \cdot$ Kensuke Sakai $^{1} \cdot$ Takeshi Makabe $^{1} \cdot$ Wataru Yamagami ${ }^{1} \cdot$ Nobuyuki Susumu $^{1,6}$. \\ Kaori Kameyama ${ }^{7} \cdot$ Kenjiro Kosaki $^{3} \cdot$ Daisuke Aoki $^{1}$
}

Received: 29 June 2020 / Accepted: 22 September 2020 / Published online: 9 October 2020

(c) The Author(s) 2020

\begin{abstract}
There has been a rapid advance in germline multigene panel testing by next-generation sequencing, and it is being widely used in clinical settings. A 56-year-old woman suspected of having Lynch syndrome was identified as a BRCA2 pathogenic variant carrier by multigene panel testing. The patient was diagnosed with endometrial cancer at the age of 39 years, and total laparoscopic hysterectomy and bilateral salpingectomy were performed at the age of 49 years; however, bilateral oophorectomy was not performed at that time. As she had a family history of colorectal cancer and a history of endometrial cancer, Lynch syndrome was suspected. However, germline multigene panel testing revealed a pathogenic BRCA2 variant rather than pathogenic variants in mismatch repair genes. In this case, with conventional genetic risk assessment, we were unable to determine whether the patient had a high risk of hereditary breast and ovarian cancer; thus, germline multigene panel testing may provide valuable information to improve disease management strategies for patients in clinical settings. Particularly, germline multigene panel testing may be useful for detecting hereditary tumor syndromes if a patient does not present with a typical family history of cancer.
\end{abstract}

Keywords BRCA2 $\cdot$ Hereditary breast and ovarian cancer $\cdot$ Multigene panel testing $\cdot$ Genetic counseling $\cdot$ Lynch syndrome

Akira Hirasawa

hir-aki45@umin.org

1 Department of Obstetrics and Gynecology, Keio University School of Medicine, Tokyo, Japan

2 Department of Clinical Genomic Medicine, Graduate School of Medicine, Dentistry and Pharmaceutical Sciences, Okayama University, 2-5-1 Shikata-cho, Kita-ku, Okayama 700-8558, Japan

3 Center for Medical Genetics, Keio University School of Medicine, Tokyo, Japan

4 Oncogene Research Unit/Cancer Prevention Unit, Tochigi Cancer Center Research Institute, Tochigi, Japan

5 Department of Genetic Medicine and Services, National Cancer Center Hospital, Tokyo, Japan

6 Department of Obstetrics and Gynecology, International University of Health and Welfare, Chiba, Japan

7 Department of Pathology, Showa University Northern Yokohama Hospital, Kanagawa, Japan

\section{Introduction}

During recent years, there has been a rapid advance in genetic testing techniques such as next-generation sequencing, and they are being increasingly used in clinical practice, rather than conventional single-gene analysis [1]. Multigene panel testing by next-generation sequencing enables simultaneous analysis of multiple genes of interest at a lower cost than conventional techniques [2-5]. In some cases, this tool has provided valuable information that has enabled to change clinical management strategies for patients [6]. Here, we present a case of an individual suspected of having Lynch syndrome, but multigene panel testing revealed that the patient was a carrier of a $B R C A 2$ pathogenic variant. 


\section{Case report}

\section{Clinical history}

The patient, who had no particular history, was diagnosed with stage Ia endometrial cancer (FIGO 1988) when she was 39 years old. Her height was $158 \mathrm{~cm}$ and weight was $56 \mathrm{~kg}$; the body mass index (BMI) was 22.4 . The histological subtype was endometrioid carcinoma Grade 1 . As the patient was never pregnant and hoped to preserve her fertility, medroxyprogesterone acetate therapy was carried out, instead of hysterectomy. As her tumor recurred when she was 49 years old, as revealed by the cytological analysis (Fig. 1), total laparoscopic hysterectomy and bilateral salpingectomy were performed, but the bilateral ovaries were not resected according to her wish. The final histopathological diagnosis was endometrioid carcinoma Grade 1 with no myometrial invasion (Fig. 2). Figure 3 shows the patient's family tree. On the maternal side, six of her uncles and aunts (II-3, 4, 5, 6, 7, and 10), as well as her grandfather (I-3), had a history of colorectal cancer. In addition, one of her maternal cousins (III-8) had ovarian cancer and a paternal cousin (III-1) had prostate cancer.

\section{Genetic counseling}

The attending physician suggested hereditary involvement, and genetic counseling was provided based on informed consent when she was 56 years old. First, she was suspected of having Lynch syndrome because of her history of endometrial cancer and family history of colorectal cancer. However, we suspected Lynch syndrome, as she fulfilled neither

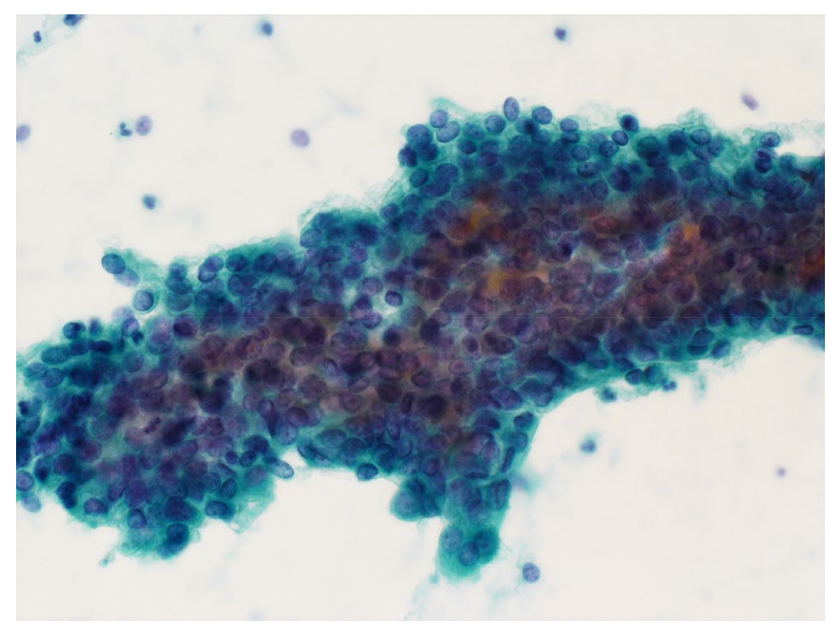

Fig. 1 Cytology of the endometrium. Cell clusters exfoliated from the endometrium showed considerable nuclear overlapping. The nuclei were irregular and hyperchromatic and had coarse chromatin. Based on these findings, endometrioid adenocarcinoma was suspected

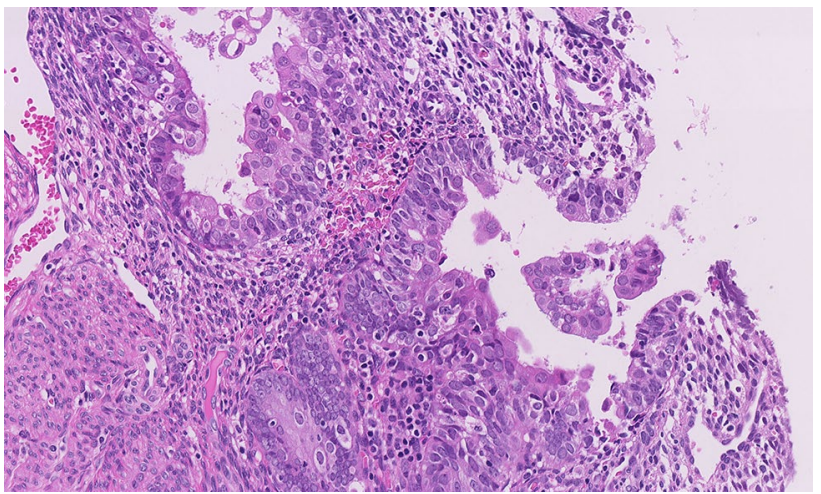

Fig. 2 Microscopic analysis of the endometrial specimen stained with Hematoxylin and Eosin. The endometrial glands showed a small nonvillous papillary architecture and were lined by cuboidal or columnar cells with pale eosinophilic cytoplasm. The nuclei were round and enlarged, without appreciable atypia

the Amsterdam criteria II nor revised Bethesda guidelines; moreover, she was not affected by colorectal cancer, and her first-degree relatives (specifically, her mother) had no history of related tumors. Thus, this patient did not show typical features of Lynch syndrome, although a genetic factor was assumed to be functional in the background. Therefore, germline panel testing using OncoGuide NCC Oncopanel System FC v. 1.0 (Table 1) (Agilent, Tokyo, Japan) was performed in addition to microsatellite instability testing and mismatch repair protein immunohistochemistry of endometrial specimens.

\section{Results}

The stability of microsatellites and the expression of mismatch repair proteins were not decreased, according to microsatellite instability testing and immunohistochemistry analysis, respectively (Fig. 4). Germline multigene panel testing revealed a $B R C A 2$ pathogenic variant (exon13: c.C6952T, p.R2318X).

\section{Clinical management}

The patient had a $B R C A 2$ pathogenic variant, and information regarding the future risk of breast, ovarian, and pancreatic cancers was collected. The option of prophylactic resection of either the mammaries (by risk-reducing mastectomy) or ovaries was recommended according to management guidelines, such as those from the National Comprehensive Cancer Network. Genetic counseling for other family members considered to be at risk was also considered. This patient is currently 56 years old, and to date, she has not undergone prophylactic surgery. Instead, she is under 


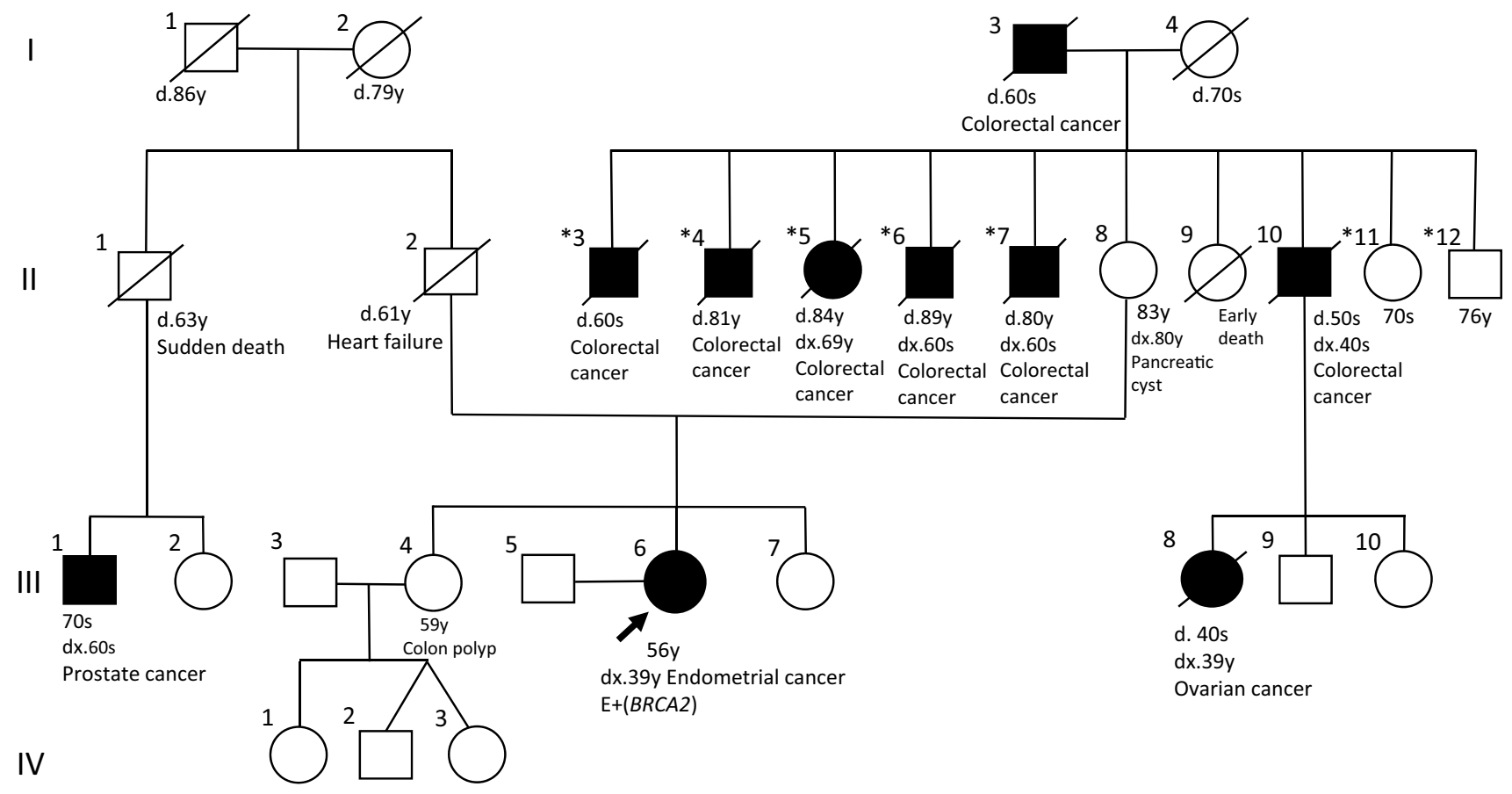

Fig. 3 Family tree. The patient in this case is indicated with an arrow. The black squares and circles indicate family members with a history of malignancy. d., age at death; dx., age at diagnosis. II-3, -7, -11, and -12 have two children each, and II-4, -5, and -6 have three children each, none of them were affected by any related tumors (data not shown)

Table 1 List of 121 genes analyzed using the NCC oncopanel system FC v. 1.0

\begin{tabular}{|c|c|c|c|c|c|c|c|c|c|c|}
\hline$A I P$ & $B R C A 1$ & $C Y L D$ & $F A N C A$ & GALNT12 & MITF & $P A L L D$ & PTCH2 & $S D H A$ & $T G F B R 2$ & $X R C C 2$ \\
\hline$A K T 1$ & $B R C A 2$ & $D D B 2$ & $F A N C B$ & GEN1 & $M L H 1$ & PHOX $2 B$ & PTEN & $S D H A F 2$ & TMEM127 & \\
\hline$A L K$ & $B R I P 1$ & DICERI & FANCC & GOLGA5 & $M L H 3$ & PIK $3 C A$ & $R A D 50$ & $S D H B$ & TP53 & \\
\hline$A P C$ & $C D C 73$ & $E G F R$ & $F A N C D 2$ & GREMI & $M R E 11 A$ & $P M S 2$ & $R A D 51 B$ & $S D H C$ & TP53BP1 & \\
\hline ATM & $\mathrm{CDHI}$ & EGLN1 & FANCE & HOXB13 & MSH2 & POLD1 & $R A D 51 C$ & $S D H D$ & TSC1 & \\
\hline$A T R$ & $C D K 4$ & $E L A C 2$ & FANCF & $K I F I B$ & MSH3 & POLE & $R A D 51 D$ & SLX4 & $T S C 2$ & \\
\hline AXINI & $C D K N 1 B$ & EPCAM & FANCG & KIT & MSH6 & POLH & $R B 1$ & SMAD4 & TSHR & \\
\hline AXIN2 & $C D K N 2 A$ & $E R B B 2$ & FANCI & $K R A S$ & MUTYH & $P P M 1 D$ & $R B M 15$ & SMARCB1 & $V H L$ & \\
\hline$B A P 1$ & CHEK1 & ESR1 & FANCL & $M A X$ & $N B N$ & PRKARIA & RECQL4 & SMARCE1 & $W R N$ & \\
\hline$B A R D 1$ & CHEK2 & EXT1 & $F A N C M$ & $M C 1 R$ & $N F 1$ & $P R K D C$ & $R E T$ & STK11 & $W T 1$ & \\
\hline$B L M$ & CTNNA1 & EXT2 & $F H$ & MEN1 & $N F 2$ & PRSS1 & $R H B D F 2$ & $S U F U$ & $X P A$ & \\
\hline$B M P R 1 A$ & CTNNB1 & FAM175A & $F L C N$ & $M E T$ & PALB2 & РTCH1 & $R N F 139$ & $T E R T$ & $X P C$ & \\
\hline
\end{tabular}

surveillance for breast, ovarian, and pancreatic cancers in our hospital.

\section{Discussion}

In this study, a $B R C A 2$ pathogenic variant was detected in the patient. The BRCA2 exon 13 variant c.C6952T, p.R2318X was identified as a pathogenic variant according to previous studies [7-9]. The frequency of this variant has been reported to be $0.44 \%$ in Japanese patients with breast cancer [8], and this variant has also been reported in Japanese patients with ovarian cancer [9]. Here, when reviewing the family tree (Fig. 3) with this genetic information, it was apparent that one maternal cousin (III-8) had a history of ovarian cancer, one paternal cousin (III-1) had prostate cancer, and no family member on either side had breast cancer. As prostate cancer is also related to hereditary breast and ovarian cancer (HBOC), it is difficult to determine the family member from whom the BRCA2 variant was derived. Additional examination in other 
Fig. 4 Immunohistochemical staining of mismatch repair proteins. The tumor cell nuclear expression of MLH1, MSH6, MSH2, and PMS2 was similar to that in the surrounding stromal cells

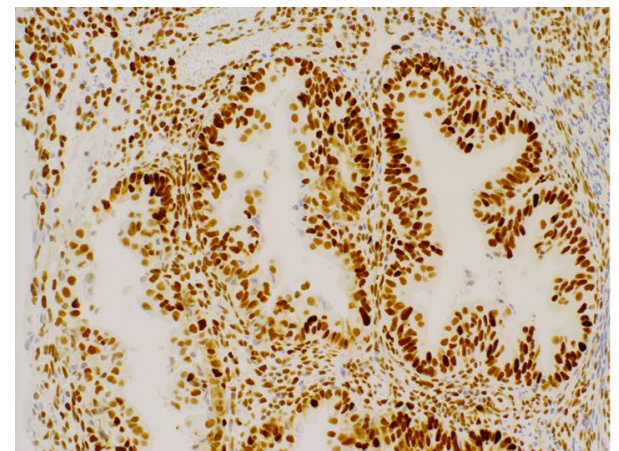

(a) MLH1

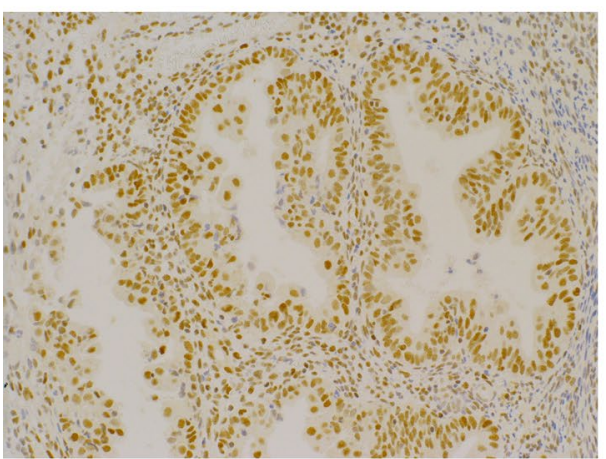

(c) $\mathrm{MSH} 2$

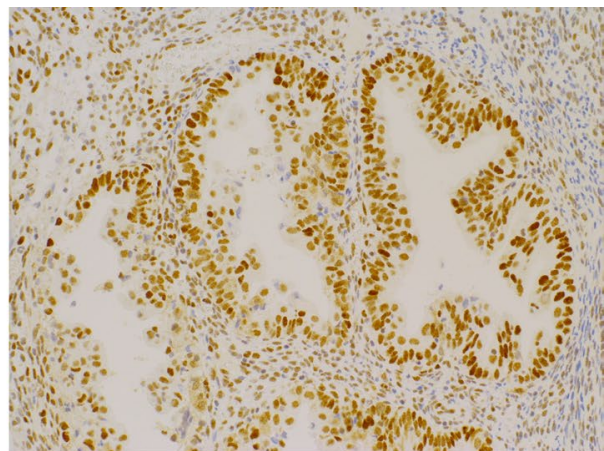

(b) MSH6

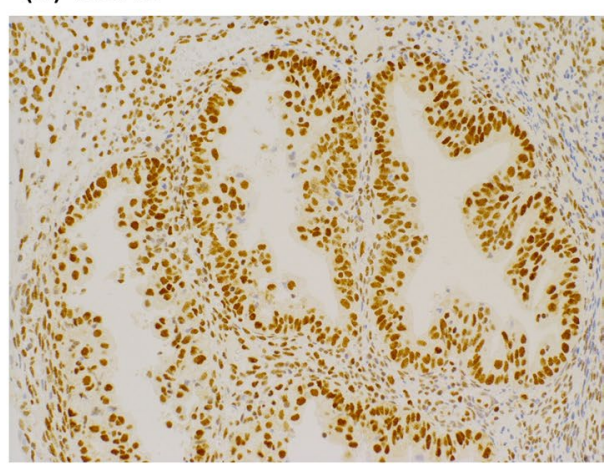

(d) PMS2 family members should be considered to clarify hereditary tumor involvement and perform segregation analysis.

One of the most important clinical considerations in this case was that the bilateral ovaries of the patient were not resected. In addition, the fallopian tubes were not pathologically examined in accordance with the SEE-FIM protocol at that time. When total laparoscopic hysterectomy and bilateral salpingectomy were performed to treat her endometrial cancer, there was no information regarding her $B R C A 2$ pathogenic variant. On the contrary, this patient did not meet the BRCA1/2 testing criteria of the National Comprehensive Cancer Network Guidelines (v. 3, 2019), as she had no history of breast or ovarian cancer and only one of her cousins had ovarian cancer. Therefore, it was difficult to evaluate the risk of $\mathrm{HBOC}$ in this patient using only the personal and family histories. In contrast, if multigene panel testing had been performed before surgery, this patient could have opted bilateral salpingo-oophorectomy based on the future ovarian cancer risk; moreover, the fallopian tubes should have been appropriately examined.

Thus, multigene panel testing may change disease management in clinical settings based on results that cannot be obtained from either conventional risk assessments or single-gene analysis. Indeed, previous studies have indicated that multigene panel testing can increase the detection rate of any pathogenic variant (including non-BRCA1/2 variants, such as Lynch syndrome genes) in patients suspected with HBOC, and this can alter clinical management strategies for cancers $[6,10]$. Kurian et al. also reported that BRCA1/2only testing is being replaced by multiple-gene sequencing for patients with breast cancer [11]. Furthermore, some previous studies identified $B R C A 2$ pathogenic variants in patients with colorectal cancer based on the age at diagnosis or family history of colorectal cancers $[12,13]$. Here, we suggest that multigene panel testing is also useful for improving the clinical management strategies of patients who had been primarily suspected to have Lynch syndrome and not HBOC.

However, several issues should be considered when performing multigene panel testing. For example, there may be some differences in the targeted genes or variant annotations among the commercially available tests. The possibility of identifying variants of unknown significance or variants in genes that are not thought to be clinically actionable should also be considered. Therefore, single gene analysis could be considered for some hereditary tumors, which have characteristic phenotypes, such as, Peutz-Jeghers syndrome. Individuals providing genetic counseling should be familiar with updated information regarding the problems mentioned above.

Regarding the relationship between the BRCAl/2 variant and endometrial cancer, Shu et al. reported that the risk of serous/serous-like endometrial carcinoma increased in $B R C A l$ variant-positive women [14]. In our case, the patient had a BRCA2 pathogenic variant, and the endometrial cancer histology was endometrioid carcinoma. Therefore, it is 
unclear whether the $B R C A 2$ variant affected the etiology of endometrial cancer. On the contrary, this patient did not have typical clinical features of endometrial cancer such as obesity or a history of diabetes mellitus. Although nulliparity could be a risk factor, it is obscure how the patient developed endometrial cancer in her thirties.

In conclusion, we present a case in which multigene panel testing revealed a $B R C A 2$ pathogenic variant in a patient who had been suspected to have Lynch syndrome, rather than HBOC. Clinicians should take detailed history of patients and their families, particularly when planning a surgery and should carefully choose an appropriate genetic testing tool that may confirm or alter the clinical management strategy.

Acknowledgements We are grateful to Ms. Kumiko Misu for assisting with genetic counseling. We also thank Ms. Atsuko Fukushima, Ms. Tomomi Noda, and Ms. Keiko Abe for administrative assistance.

Author contributions AH designed the study and TY wrote the initial draft of the manuscript. KS contributed to the genetic analysis and interpretation of data. KK reviewed the pathology and cytology. All other authors contributed to the collection of data. All authors approved the final version of the manuscript and agree to be accountable for all aspects of the work in ensuring that questions related to the accuracy or integrity of any part of the work are appropriately investigated and resolved.

Funding This study was partially funded by the JSPS Bilateral program and JSPS KAKENHI (Grant numbers 17K19611 and 18K09298); the National Cancer Center Research and Development Fund (Grant number 28-A-1); Practical Research for Innovative Cancer Control; and Program for Promoting Practical Applications of Genomic Medicine from the Japan Agency for Medical Research and Development, AMED (Grant numbers 15ck0106097 h0102 and 15cK0106168 h0201), and Health Labour Sciences Research Grant (20EA1027).

\section{Compliance with ethical standards}

Conflict of interest The authors declare that they have no conflict of interest.

Ethical approval This study was approved by the ethics committee of Keio University Hospital (20070081, 20130393, 20160391).

Human participants and/or animals This study was performed in accordance with the Declaration of Helsinki.

Informed consent Informed consent was obtained from the client included in the study.

Open Access This article is licensed under a Creative Commons Attribution 4.0 International License, which permits use, sharing, adaptation, distribution and reproduction in any medium or format, as long as you give appropriate credit to the original author(s) and the source, provide a link to the Creative Commons licence, and indicate if changes were made. The images or other third party material in this article are included in the article's Creative Commons licence, unless indicated otherwise in a credit line to the material. If material is not included in the article's Creative Commons licence and your intended use is not permitted by statutory regulation or exceeds the permitted use, you will need to obtain permission directly from the copyright holder. To view a copy of this licence, visit http://creativecommons.org/licenses/by/4.0/.

\section{References}

1. Swisher EM (2015) Usefulness of multigene testing: catching the train that's left the station. JAMA Oncol 1:951-952

2. LaDuca H, Stuenkel AJ, Dolinsky JS et al (2014) Utilization of multigene panels in hereditary cancer predisposition testing: analysis of more than 2000 patients. Genet Med 16:830-837

3. Tung N, Battelli C, Allen B et al (2015) Frequency of mutations in individuals with breast cancer referred for $B R C A 1$ and $B R C A 2$ testing using next-generation sequencing with a 25 -gene panel. Cancer 121:25-33

4. Walsh T, Lee MK, Casadei S et al (2010) Detection of inherited mutations for breast and ovarian cancer using genomic capture and massively parallel sequencing. Proc Natl Acad Sci USA 107:12629-12633

5. Walsh T, Casadei S, Lee MK et al (2011) Mutations in 12 genes for inherited ovarian, fallopian tube, and peritoneal carcinoma identified by massively parallel sequencing. Proc Natl Acad Sci USA 108:18032-18037

6. Desmond A, Kurian AW, Gabree M et al (2015) Clinical actionability of multigene panel testing for hereditary breast and ovarian cancer risk assessment. JAMA Oncol 1:943-951

7. Sugano K, Nakamura S, Ando J et al (2008) Cross-sectional analysis of germline BRCA1 and BRCA2 mutations in Japanese patients suspected to have hereditary breast/ovarian cancer. Cancer Sci 99:1967-1976

8. Momozawa Y, Iwasaki Y, Parsons MT et al (2018) Germline pathogenic variants of 11 breast cancer genes in 7051 Japanese patients and 11,241 controls. Nat Commun 9:403

9. Hirasawa A, Imoto I, Naruto T et al (2017) Prevalence of pathogenic germline variants detected by multigene sequencing in unselected Japanese patients with ovarian cancer. Oncotarget 8:112258-112267

10. Eoh KJ, Kim JE, Park HS et al (2018) Detection of germline mutations in patients with epithelial ovarian cancer using multi-gene panels: beyond BRCA1/2. Cancer Res Treat 50:917-925

11. Kurian AW, Ward KC, Hamilton AS et al (2018) Uptake, results, and outcomes of germline multiple-gene sequencing after diagnosis of breast cancer. JAMA Oncol 4:1066-1072

12. Pearlman R, Wendy LF (2017) Prevalence and spectrum of germline cancer susceptibility gene mutations among patients with early-onset colorectal cancer. JAMA Oncol 3:464-471

13. Garre P, Martín L, Sanz J et al (2015) BRCA2 gene: a candidate for clinical testing in familial colorectal cancer type X. Clin Genet 87:582-587

14. Shu CA, Pike MC, Jotwani AR et al (2016) Uterine cancer after risk-reducing salpingo-oophorectomy without hysterectomy in women with BRCA mutations. JAMA Oncol 2:1434-1440

Publisher's Note Springer Nature remains neutral with regard to jurisdictional claims in published maps and institutional affiliations. 